\title{
Prophylaktische Mastektomie und Rekonstruktion: Wie ist das Körperbild?
}

Frauen, die sich für eine prophylaktische Mastektomie mit Rekonstruktion der Brust entscheiden, sind damit meist sehr zufrieden. Es gibt aber auch Hinweise, dass das Körperbild darunter leidet.

\section{- rauen mit genetisch bedingt stark er- höhtem Brustkrebsrisiko wird heute vielfach eine prophylaktische Mastekto- mie mit plastischer Rekonstruktion ange- boten. Mariska den Heijer et al. haben prospektiv untersucht, wie sich ein solcher Eingriff auf das Körperbild auswirkt. \\ Dazu wurden 36 Frauen vor und sechs Monate sowie sechs bis neun Jahre nach}

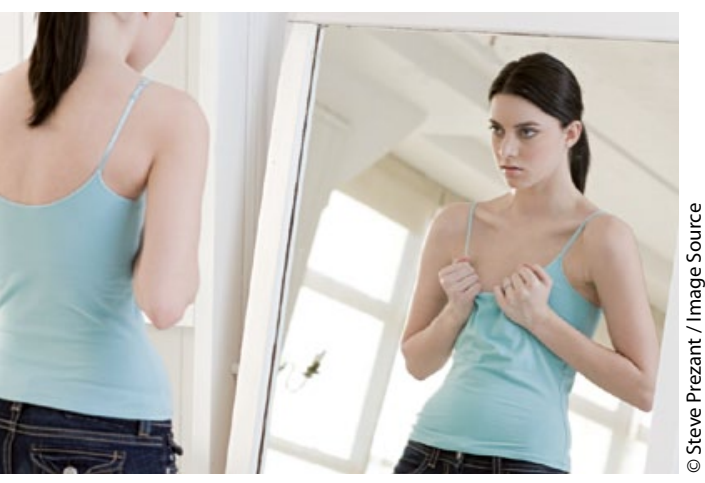

Ängste und Stress nehmen ab, aber das Körperbild kann leiden. dem Eingriff befragt. Allgemeine Ängste und Stress wurden mit der Hospital Anxiety and Depression Scale (HADS, 0-42 Punkte) erfasst. Hier war der Wert mit im Schnitt 10 Punkten vor der Op. deutlich erhöht, sank danach auf 7,5 und nach einigen Jahren auf 6,6 Punkte. Werte bis 7 Punkte gelten als normal. Brustkrebsspezifische Ängste wurden mit der Impact of Event Scale (IES, 0 bis 75 Punkte) erfasst, auf der der Wert von knapp 23 Punkten vor der Op. auf 13 Punkte nach sechs Monaten und 6,1 Punkte nach sechs bis neun Jahren zurückging. Insgesamt verschwanden also allgemeine wie Brustkrebs-spezifische Ängste sowie der Stress.

Anders sah es mit dem Körperbild aus. Wurden die Frauen nach ihrer Attraktivität, Weiblichkeit und ihrem Aussehen befragt, so waren die Werte vor der Op. deutlich besser. Auf einer Skala von 5 bis 25 Punkten beurteilten sie sich vor der Operation im Schnitt mit 10,7 Punkten. Der Wert verschlechterte sich sechs Mo- nate nach dem Eingriff auf 12,4 Punkte und fiel nach sechs bis neun Jahren wieder leicht auf 11,7 Punkte. Auch mit Aussehen und Gefühl ihrer Brüste waren sie vor der Op. deutlich zufriedener, auch das besserte sich nach einigen Jahren etwas.

Dabei hatten Frauen, die sich vor der Op. als sehr attraktiv bewertet hatten, hinterher weniger Probleme mit ihrem Aussehen als Frauen, die schon zuvor mit ihrem Äußeren unzufrieden gewesen waren. Sie brauchen möglicherweise eine besondere psychologische Unterstützung, wenn sie sich für eine präventive Mastektomie entscheiden.

Fazit: Entscheiden sich Frauen mit hohem Risiko für eine präventive Mastektomie, nehmen anschließend Ängste und Stress ab. Der Preis dafür: Sie fühlen sich häufig weniger attraktiv.

Thomas Müller

den Heijer $M$ et al. Body image and psychological distress after prophylactic mastectomy and breast reconstruction in genetically predisposed women: A prospective longterm follow-up study. Eur J Cancer. 2012;48(9):1263-8.

\section{Sport schützt auch in der Postmenopause}

\section{Regelmäßige körperliche Aktivität in der Freizeit ist auch zur Prävention von Brustkrebs geeignet. US-Epidemiologen gingen jetzt der Frage nach, in welchem Lebensabschnitt sie etwas bringt und wie intensiv sie sein muss.}

$\mathrm{R}$ egelmäßige körperliche Aktivität senkt das Krebsrisiko, auch das Risiko für Brustkrebs. Unklar war bisher, auf welche Lebensphasen und welche Intensität körperlicher Aktivität es dabei vor allem ankommt. Epidemiologen von der Universität von North Carolina in Chapel Hill gingen dem im Long Island Breast Cancer Study Project mit etwa 1.500 Brustkrebspatientinnen und mehr als 1.550 Frauen ohne Brustkrebs als Kontrollen nach. Es handelt sich dabei um eine soziodemographisch recht homogene Population von Frauen zwischen 20 und 98 Jahren, die allerdings nicht dem Querschnitt der US-Bevölkerung entspricht. Die meisten für die Studie befragten Frauen hatten mindestens ein Kind.
Wie die Wissenschaftler berichten, spielt die Intensität der körperlichen Aktivität, gemessen in MET (metabolische Äquivalente), und deren Dauer mit Blick auf die Risikoreduktion keine Rolle. Im Vergleich zu nicht regelmäßig körperlich aktiven Frauen hatten jene Frauen in der Postmenopause das geringste Brustkrebsrisiko, die sagten, sie seien zwischen der Geburt des ersten Kindes und der Menopause mit 10 bis 19 Stunden pro Woche regelmäßig körperlich aktiv gewesen: Ihr Brustkrebsrisiko war um mehr als 30\% verringert (Odds Ratio [OR] 0,67).

Selbst Frauen, die erst ab der Menopause sportlich aktiver wurden, profitierten davon mit einer OR von 0,70 bei neun bis 17 Stunden Aktivität pro Woche.
Frauen, die trotz regelmäßiger körperlicher Aktivität mindestens drei Kilogramm Gewicht zulegten, machten damit die erhoffte Krebsprävention zunichte, vor allem, wenn die Gewichtszunahme nach der Menopause erfolgte. Das weist in die Richtung der Ergebnisse einer Metaanalyse, wonach Frauen, die trotz regelmäBiger körperlicher Aktivität einen Body Mass Index (BMI) über $30 \mathrm{~kg} / \mathrm{m}^{2}$ haben, ihr Brustkrebsrisiko nicht senken können.

Fazit: Frauen profitieren von regelmäBiger körperlicher Aktivität nicht nur im gebärfähigen Alter, sondern auch noch nach der Menopause: Mit 10 bis 19 Stunden pro Woche lässt sich das Brustkrebsrisiko um 30\% reduzieren, vorausgesetzt, die Frau nimmt nicht zu. Peter Leiner

McCullough LE et al. Fat or fit: The joint effects of physical activity, weight gain, and body size on breast cancer risk. Cancer. 2012 Jun 25 [Epub ahead of print]. 